\title{
Bone marrow-derived pancreatic stellate cells in rats
}

Gisela Sparmann ${ }^{1, *}$, Marie-Luise Kruse ${ }^{2, *}$, Nicole Hofmeister-Mielke ${ }^{1,3}$, Dirk Koczan ${ }^{4}$, Robert Jaster ${ }^{1}$, Stefan Liebe ${ }^{1}$, Daniel Wolff ${ }^{1,3}$, Jörg Emmrich ${ }^{1}$

${ }^{I}$ Division of Gastroenterology, Department of Internal Medicine, University of Rostock, 18057 Rostock, Germany; ${ }^{2}$ Laboratory for Molecular Gastroenterology and Hepatology, Department of General Internal Medicine, UKSH Campus Kiel, 24105 Kiel, Germany; ${ }^{3}$ Department of Hematology and Internal Oncology, University Hospital Regensburg, 93503 Regensburg, Germany; ${ }^{4}$ Institute of Immunology, University of Rostock, 18057 Rostock, Germany

Origin and fate of pancreatic stellate cells (PSCs) before, during and after pancreatic injury are a matter of debate. The crucial role of PSCs in the pathogenesis of pancreatic fibrosis is generally accepted. However, the turnover of the cells remains obscure. The present study addressed the issue of a potential bone marrow (BM) origin of PSCs. We used a model of stable hematopoietic chimerism by grafting enhanced green fluorescence protein (eGFP)-expressing BM cells after irradiation of acceptor rats. Chimerism was detected by FACS analysis of eGFP-positive cells in the peripheral blood. Dibutyltin dichloride (DBTC) was used to induce acute pancreatic inflammation with subsequent recovery over 4 weeks. Investigations have been focused on isolated cells to detect the resting PSC population. The incidence of eGFP-positive PSC obtained from the pancreas of chimeric rats was approximately $7 \%$ in healthy pancreatic tissue and increased significantly to a mean of $18 \%$ in the restored pancreas 4 weeks after DBTC-induced acute inflammation. Our results suggest that BM-derived progenitor cells represent a source of renewable stellate cells in the pancreas. Increased numbers of resting PSCs after regeneration point toward enhanced recruitment of BM-derived cells to the pancreas and/or re-acquisition of a quiescent state after inflammation-induced activation.

Keywords: bone marrow, rat, pancreas, regeneration

Cell Research (2010) 20:288-298. doi:10.1038/cr.2010.10; published online 26 January 2010

\section{Introduction}

There is consensus regarding the crucial role of pancreatic stellate cells (PSCs) in the pathogenesis of pancreatic fibrosis. These specialized fibroblasts are defined by the presence of storage deposits of fat droplets with vitamin A and by a transition from a quiescent status into activated myofibroblast cells during pancreatic injury in vitro after prolonged cultivation $[1,2]$. Because of the clinical relevance, the molecular mechanisms of the activation process are a matter of intensive investigation [3]. In contrast, the turnover of PSCs remains obscure.

\footnotetext{
*These two authors contributed equally to this work. Correspondence: Gisela Sparmann

Tel: + 49 (0) 381-494-7569; Fax: + 49 (0) 381-494-7482

E-mail: gisela.sparmann@med.uni-rostock.de

Abbreviations: $\alpha$-SMA ( $\alpha$-smooth muscle actin); BM (bone marrow); DBTC (dibutyltin dichloride); eGFP (enhanced green fluorescence protein); PSC (pancreatic stellate cells)

Received 21 August 2009; revised 28 October 2009; accepted 11 November 2009; published online 26 January 2010
}

Removal of unwanted cells is thought to occur by apoptosis [4]. However, the source of quiescent PSCs in the restored pancreas, subsequent to a pancreatic injury, has not been investigated so far.

Previous studies concerning pancreatic regeneration from stem cell sources have concentrated on the substitution of $\beta$-cells in the endocrine islets of Langerhans. Reports regarding the potential origin of insulin-producing cells are extremely controversial, including bone marrow (BM)-derived progenitors, pancreas-resident stem cells, transdifferentiation of acinar as well as duct cells and self-duplication of adult $\beta$-cells [5-10]. Recently, Mato et al. [11] reported an in vitro-selected mitoxantrone-resistant cell pool from rat pancreas displaying characteristics of PSCs. These cells were reported to differentiate into insulin-producing cells upon adequate stimulation.

The contribution of BM-derived cells to myofibroblasts and fibrogenic processes has been reported by various authors. Russo et al. [12] suggested that the BM is a major source of myofibroblasts in chronic liver injury. Lama and Phan [13] provided evidence for an extrapulmonary 
origin of fibroblasts in the fibrotic lung. Scar formation following a myocardial infarction has been shown to be associated with the appearance of BM-derived $\alpha$-SMApositive myofibroblasts [14]. Using a mouse model of severe chronic pancreatic injury, Marrache et al. [15] have shown that some stellate cells originated from the BM.

Although controversial, recent published data support the general conclusion that cell plasticity and migration between different organs might represent a constitutive phenomenon persisting throughout life.

In this study, we examined the contribution of BM to the PSC population. For this approach, a model of rats with a stable hematopoietic enhanced green fluorescence protein (eGFP) chimerism has been used. We investigated isolated PSCs obtained from untreated control rats as well as from the restored organ following an episode of acute pancreatitis induced by dibutyltin dichloride (DBTC). The isolation method for PSC was based upon fat content, thus addressing the quiescent PSC population [16].

We provide evidence for the existence of a resting PSC population derived from BM in rat pancreas under steady state conditions before inflammation and after regeneration, thus establishing BM as a source of quiescent PSCs.

\section{Results}

Characterization of PSC phenotype in the model of acute pancreatitis

The previous characterization of inflammatory cell infiltration during DBTC-induced acute pancreatitis was extended to the analysis of number and phenotype of PSCs [17]. For this approach, the expression of the intermediate filament desmin and the activation marker $\alpha$-smooth muscle actin ( $\alpha$-SMA) was investigated by immunohistochemistry. Unlike desmin, in the healthy organs, $\alpha$-SMA-positive cells were found almost exclusively around blood vessels and pancreatic ducts (Figure $1 \mathrm{~A}, \mathrm{a}, \mathrm{d}$ and 1B). During the first week of pancreatitis, the number of desmin-positive and $\alpha$-SMA-positive cells increased significantly, reaching a maximum at day 7 (Figure 1A, b, e and 1B). Subsequently, cells positive for each marker declined, reaching nearly normal values after 2 weeks (Figure 1A, c, f and 1B).

These results indicate that inflammatory processes induced PSC activation, including enhanced proliferation and differentiation into the myofibroblast-like phenotype. The healing process was associated with a depletion of leukocytes and a decrement of PSCs, finally leading to pancreatic restoration.
Rat model of hematopoietic eGFP chimerism

The FACS analysis of peripheral blood leukocytes obtained from transplanted BN/LEW rats revealed normal peripheral blood cell counts and a stable hematopoietic chimerism of $71 \% \pm 12 \%(n=44, P=0.05)$ between 8 weeks and 12 months after BM transplantation (Figure 2). Apart from temporary weight loss during 2-3 days after irradiation, no significant toxicity was observed due to the transplantation procedure. Furthermore, no signs of acute or chronic graft-versus-host disease were detectable at any time after transplantation.

\section{Detection of eGFP-postive cells in pancreatic tissue}

Multiphoton microscopy was employed to see whether green fluorescence would be detectable in tissue unaltered by fixation and sectioning, as well as to get an impression of tissue architecture and distribution of cells. Investigation of excorporated fresh unfixed pancreatic tissue from eGFP transgenic donor rats using multiphoton microscopy revealed bright green fluorescence from eGFP expression in pancreatic acinar cells (Figure 3A). Also, interstitial cells positioned between pancreatic lobules were seen to express eGFP (Figure 3A), though much less obviously, because of their smaller size and elongated shape. When freshly explanted, pancreatic tissue from BM-transplanted rats was analyzed, we found green cells interspersed in otherwise non-fluorescent pancreatic tissue (Figure 3B). No clues to their identity could be drawn from the shape of the eGFP-positive cells, except that these were most obviously cells derived from the BM of eGFP-expressing donor rats, which migrated into the pancreas. Furthermore, as shown in Figure $3 \mathrm{~B}$, cells were found in a non-random distribution.

We then performed confocal laser scanning microscopy (cLSM) on cryosections of pancreatic tissue. We found that green fluorescent cells in transplanted animals were situated in the interlobular septa (Figure 3C), as depicted by positive cells outlining the margin of an acinar lobule and following its curvature. Other cells were found in cell masses at random (Figure 3D) with more extended shape and cellular extensions. We were unable to find eGFP-expressing islet cells or acinar or duct cells (data not shown). Indirect immunofluorescence staining showed that some of the green fluorescent cells in pancreatic tissue were positive for desmin or $\alpha$-SMA expression (data not shown).

Characterization of in vitro propagated PSCs isolated from chimeric rats

To analyze the BM origin of non-activated PSCs in transplanted rats, investigations have been performed using isolated cells. The purification procedure based on 

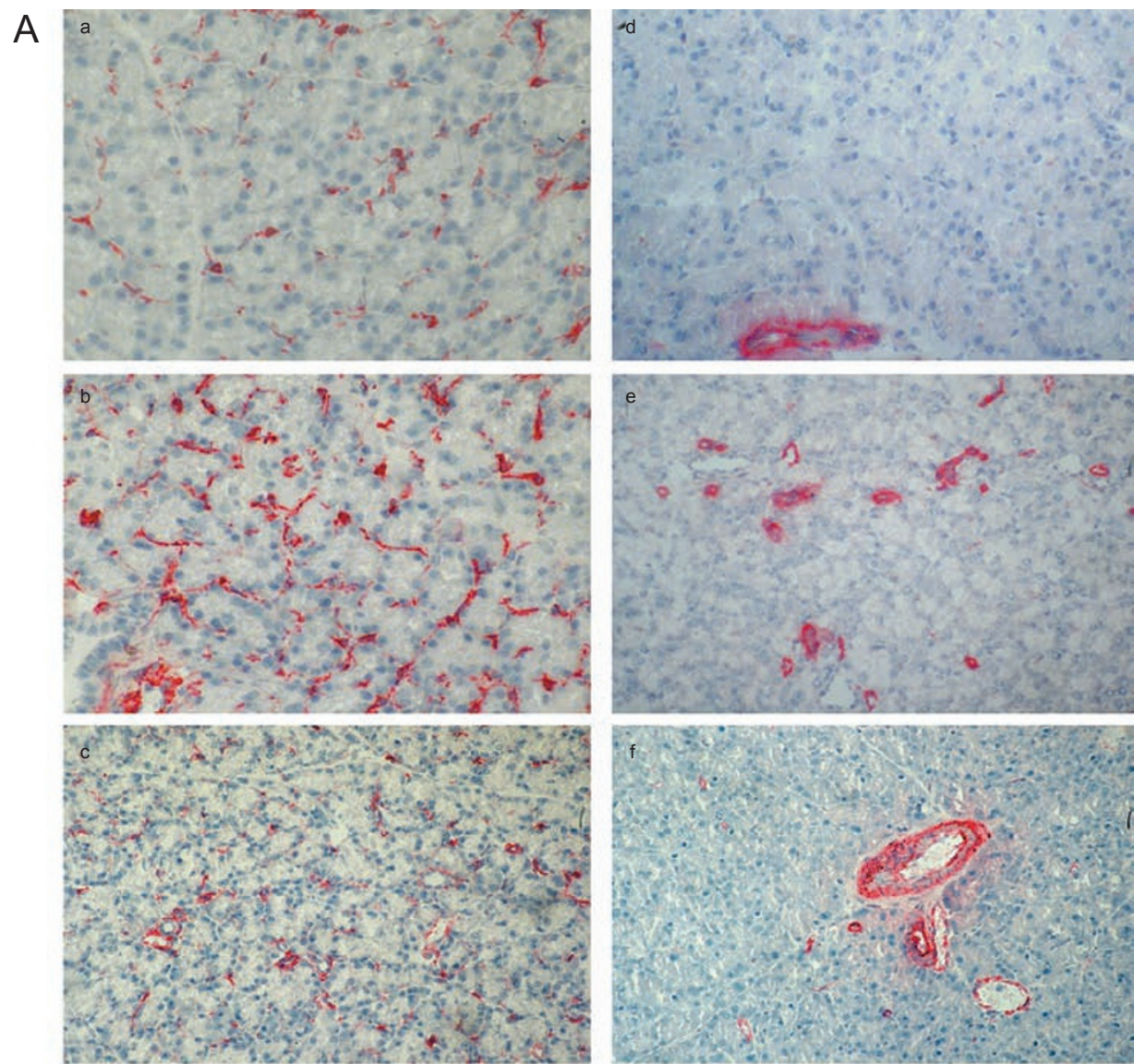

B

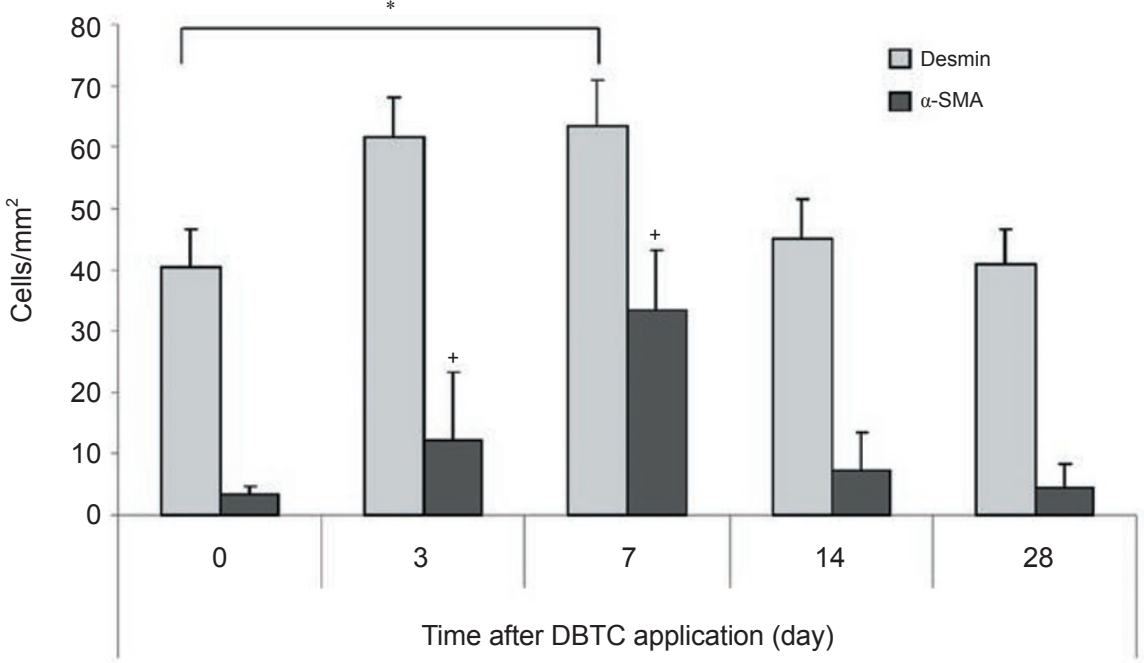

Figure 1 Immunocytochemical analysis of desmin (a-c) and $\alpha$-SMA (d-f) in pancreatic tissue obtained from controls and after the application of $4 \mathrm{mg}$ per $\mathrm{kg}$ body weight of DBTC. Cryostat sections of pancreatic tissue were incubated with a mouse monoclonal antibody detecting desmim or $\alpha$-SMA. Primary antibody binding was visualized using the APAAP technique. Cell nuclei were counterstained with hematoxylin. (A) Representative microphotographs of controls (a, d), and at 7 days (b,e) and 28 days (c, f) after DBTC application stained for desmin (a-c) and $\alpha$-SMA (d-f) (original magnification: a, b, d, e, ×200; c, f, $\times 100)$. (B) Quantitative evaluation of the expression of desmin and $\alpha$-SMA during the time course of DBTC-induced pancreatitis. At 3, 7, 14 and 28 days after i.v. injection of $4 \mathrm{mg}$ per $\mathrm{kg}$ body weight of DBTC, the pancreas was removed and subjected to the immunohistological staining procedure as described. Three pancreata of each time point were evaluated counting positive cells. Results are expressed as mean $\pm \mathrm{SEM}, * P=0.02,{ }^{+} P=0.05$ compared to controls. 

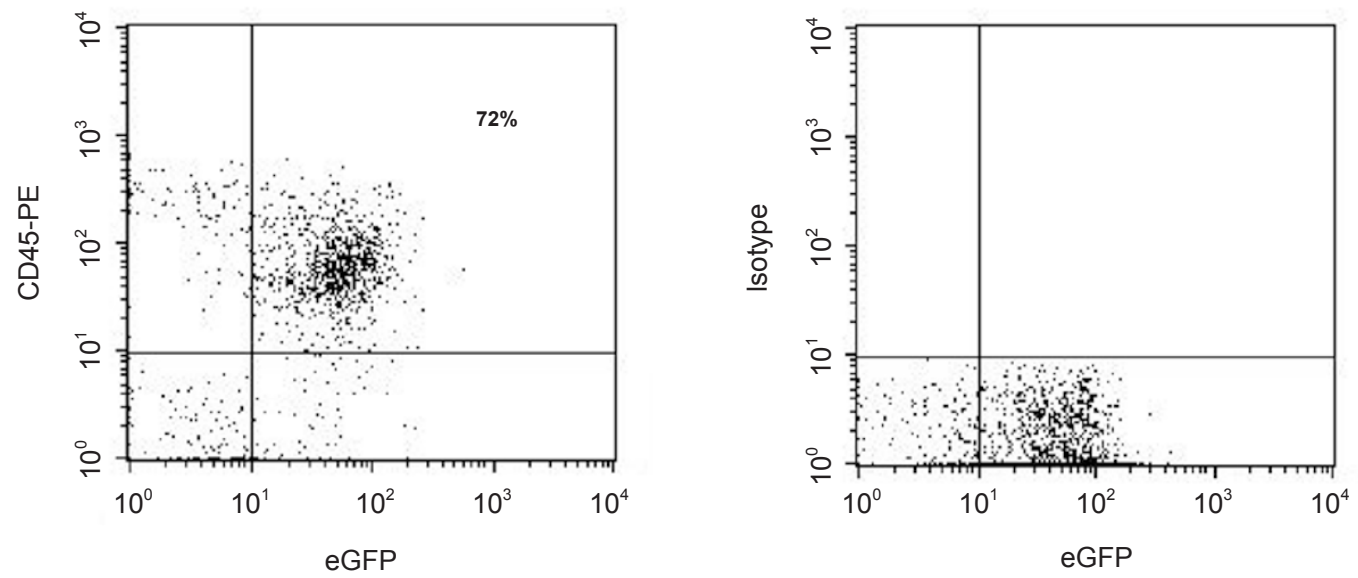

Figure 2 Representative dot plots of FACS analysis showing eGFP-expressing peripheral PBMCs obtained from rats with hematopoietic eGFP chimerism. Left panel: A PE-labeled anti-CD45 antibody was used to define leukocytes, right panel: isotype control.
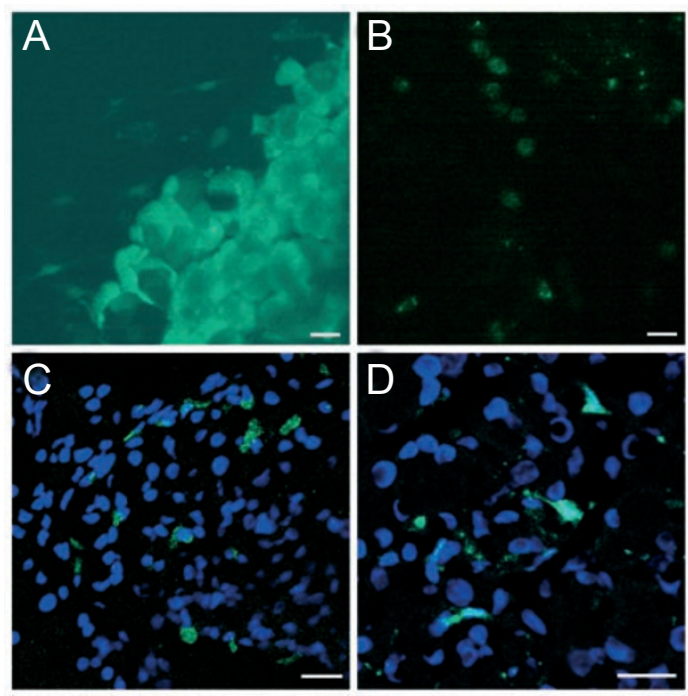

Figure 3 Analysis of endogenous eGFP fluorescence in pancreatic tissue. Multiphoton analysis (A, B) carried out on excorporated fresh pancreatic tissue of donor rats showed bright green fluorescence in pancreatic acini and interstitial cells (A). On the right, acinar cell mass of the rim of a lobule is seen, with interstitial cells of mostly elongated fibroblast-like appearance extending to the left upper region. Green fluorescence appears to be much weaker because of the reduced volume and mass of these interstitial cells. When the excorporated fresh pancreatic tissue of BM-transplanted control rats was analyzed, green fluorescence was detected in cells interspersed in otherwise non-fluorescent tissue (B). The distribution of the cells is reminiscent of interstitial areas, but did not resemble acini, islets or ductal structures. (C, D) Examination of cryosections from BMtransplanted rats by confocal laser scanning microscopy (cLSM) showed green fluorescent cells in normal pancreas (C) and at 28 days after induction of pancreatitis by DBTC (D) in otherwise non-eGFP-expressing tissue (nuclear staining: blue; scale bars represent $20 \mu \mathrm{M})$. the high fat content of quiescent PSCs ensured the nonactivated phenotype of freshly prepared stellate cells [16]. Irrespective of the previous treatment, PSCs isolated from Tx controls or from regenerated pancreas 4 weeks after DBTC application showed a typical stellate-shaped morphology (data not shown). The activation process of PSCs has been shown to be accompanied by a gradual loss of lipid droplets and rising expression of $\alpha$-SMA $[1,2,16]$. Figure $4 \mathrm{~A}$ and $4 \mathrm{~B}$ summarize the characteristic course of both markers in PSCs obtained from the restored pancreas, reflecting the typical transition from resting cells into the activated myofibroblastic phenotype during in vitro cultivation.

Figure 4C shows the expression of mRNA encoding the chemokine receptor 2 (CCR2) in PSCs at different time points after isolation. There was a moderate, but not significant, reduction of the transcript levels after long-term cultivation, indicating a constitutive expression of CCR2. This result supports the hypothesis that PSCs would be responsive to chemotactic factors that are known to be essentially involved in cell migration [18].

PSCs isolated from BM-transplanted rats at 4 weeks after the injection of $6 \mathrm{mg}$ per $\mathrm{kg}$ body weight of DBTC were grown on cover glasses for 2 days (Figure 5A, 5B, $5 \mathrm{E}, 5 \mathrm{~F}$ ) and 4 days (Figure 5C, 5D, 5G, 5H). Subsequently, specimens were analyzed for the expression of eGFP and stellate cell markers by immunofluorescence using cLSM. Figure 5 shows representative images summarizing the expression of desmin (E, G) and $\alpha$-SMA (A, C) in eGFP-positive PSCs. Desmin was found to be constitutively expressed (Figure 5E, 5G). In contrast, $\alpha$-SMA staining of stress fibers could not be detected in 2-daycultures (Figure $5 \mathrm{~A}$ ), but the activation marker was 
A
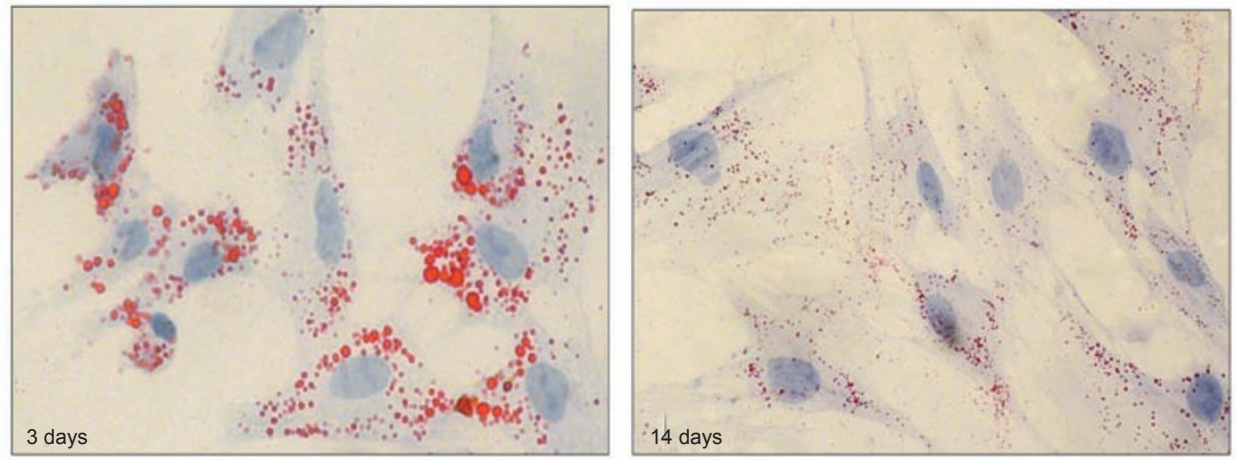

B

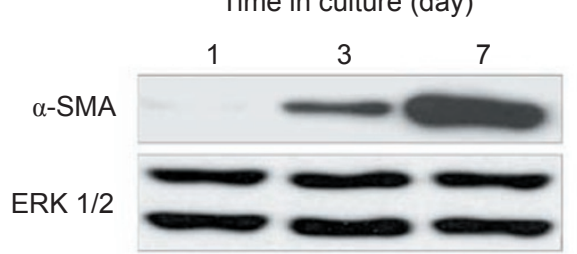

C

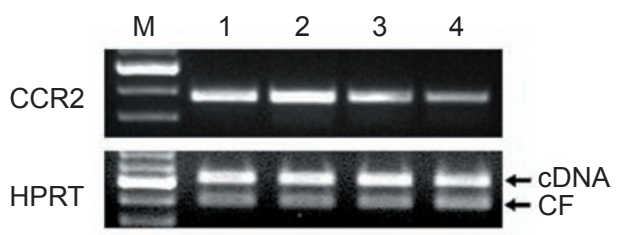

Figure 4 Characterization of PSCs isolated from regenerated pancreas. (A) Detection of lipid droplets at 3 days (left) and 14 days (right) after PSC isolation. Cells grown on glass coverslips were fixed at the indicated time point followed by staining with oil red O. Nuclei were visualized with Mayer's hematoxylin (original magnification $\times 200$ ). (B) Representative immunoblot showing the expression of $\alpha$-SMA during in vitro cultivation of PSCs. Cells were harvested 1, 3 and 7 days after isolation, protein extracts were prepared and subjected to the western blot procedure using an anti- $\alpha$-SMA antibody. To show equal protein loading, blot was re-probed with an anti-ERK 1/2 antibody. (C) Expression of mRNA encoding CCR2. Total RNA was isolated from PSCs at day 2 (lane 1), day 7 (lane 2), day 14 (lane 3) and day 21 (lane 4), transcribed into cDNA and subjected to PCR with CCR2-specific primers. Amplification products (470 bp) were electrophoretically separated in an ethidium bromidecontaining agarose gel; fluorescence intensity reflects the cDNA amount. The various cDNA samples were adjusted to equal input concentrations by competitive PCR using the housekeeping gene HPRT (608 bp); CF, internal standard (499 bp); M, 100-bp molecular weight marker.

strongly upregulated after 4 days accompanied by the formation of $\alpha$-SMA stress fibers (Figure 5C). Prolonged culture resulted in enhanced proliferation, which was clearly visible already at 4 days of culture compared to 2 days (Figure 5C, 5G versus 5A, 5E). Prolonged culture also leads to loss of eGFP expression and acquisition of a more myofibroblast-like shape with concomitantly higher expression of $\alpha$-SMA (data not shown). Our preparation procedure is dedicated to the isolation of quiescent PSCs, whereas activated cells without lipid droplets get lost. Therefore, as expected, we did not observe differences in proliferation rates of PSCs from Tx control pancreas or DBTC-treated pancreas.

\section{Analysis of eGFP transgene by protein expression and at gene level \\ PSCs isolated from Tx controls and Tx-DBTC rats were subjected to flow cytometry for the detection of the eGFP fluorescence (Figure 6). Cells were analyzed only after adherence for at least 2 days, to ensure removal of}

debris or non-PSC cells, that is, acinar cells, which tend to contain autofluorescent material interfering with fluorescence detection. The FACS analysis revealed that the fluorescence intensity decreased during the in vitro cell cultivation. This phenomenon may be attributed to a reduction of gene expression in cultivated cells. However, in all cases, the number of eGFP-positive PSCs obtained from Tx-DBTC animals was higher compared with Tx controls. Figure 6A shows representative FACS histograms of 5-day-cultured PSCs obtained from Tx control and Tx-DBTC animals. Including PSCs cultivated from 4 to 10 days, the mean value of eGFP-positive PSCs obtained from Tx-DBTC rats averaged 18\%; the mean of Tx controls was about 7\% (Figure 6B).

To exclude the fact that leukocytes contribute substantially to the eGFP fluorescence, the common leukocyte antigen CD45 was analyzed using a phycoerythrin (PE)labeled antibody. We could show that even at day 2, only about $1 \%$ of the cells were CD45 positive (Figure 6A, inset). 

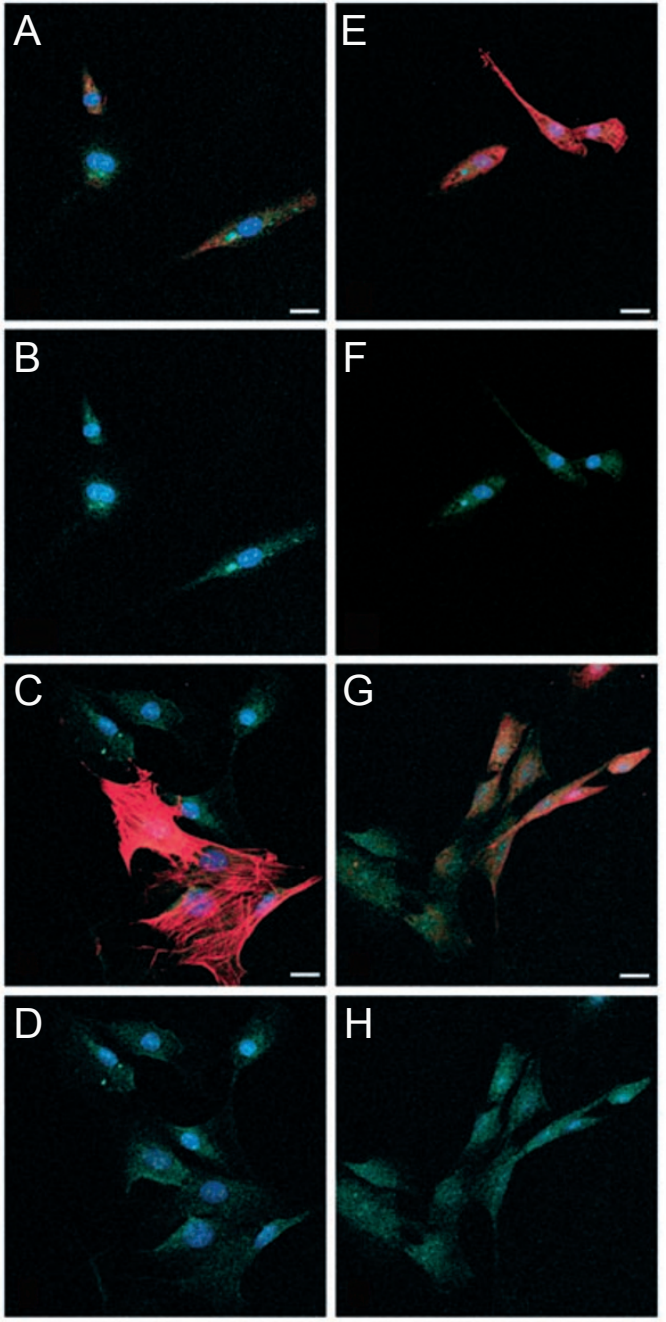

Figure 5 Analysis of endogenous eGFP fluorescence and expression of $\alpha$-SMA or desmin by confocal laser scanning microscopy. PSCs were isolated from pancreas 28 days after the induction of the acute pancreatitis by DBTC and grown for 2 days (A, B, E, F) and 4 days (C, D, G, H) followed by staining for $\alpha$-SMA (A-D) and desmin $(\mathbf{E}-\mathbf{H})$, depicted in red. Shown here are cell samples as merged pictures (A, C, E, G) and the corresponding eGFP-only fluorescence (B, D, F, H) in the identical specimen. After 2 days in culture, cells are sparse, because proliferation has not yet started and after 4 days, the number of cells increased significantly. The same applies to $\alpha$-SMA staining (A, C). After 2 days in culture, cells show no typical staining for $\alpha$-SMA (A) and after 4 days, bright red fluorescence is detected in stress fibers throughout the cells (C). Please note that the detector gain for red fluorescence was adjusted to the highest levels of expression, thus some cells appear to be negative for $\alpha$-SMA, because the expression was significantly lower. Desmin staining $(\mathbf{E}, \mathbf{G})$ was detected at high levels already after 2 days of culture (E) and remains so after 4 days of culture (G). All cells were positive for desmin, but as CLSM analysis depicts only optical slices of cells, some of the cells appear to be negative because of different planes of growth and differences in expression, as for $\alpha$-SMA. All cells investigated revealed eGFP expression as depicted by green fluorescence at these times in culture (B, D, F, H). (Scale bars represent $20 \mu \mathrm{M}$ ).
The hematopoietic eGFP chimerism in the engrafted rats constituted about $70 \%$ of eGFP-positive leukocytes. In consequence, at least $70 \%$ of the BM-derived cells in the chimeric animals should have the eGFP transgene, independent of the protein expression and/or fluorescence detection. Therefore, we determined the content of eGFP-encoding DNA in isolated PSCs using a real-time PCR approach. Results are summarized in Figure 7. We found that PSCs, isolated from control animals with a stable hematopoietic eGFP chimerism, contained eGFPpositive cells as shown by the presence of the eGFP transgene in total DNA. The percentage of eGFP-positive PSCs obtained from the restored organ subsequent to DBTC pancreatitis was about threefold higher compared to Tx controls, as detected by PCR amplification of the eGFP transgene.

\section{Discussion}

The current study addresses the issue of the contribution of BM cells to the origin of PSCs. BM-derived myofibroblasts have been reported in various organs [12-14]. In contrast, little is known regarding the origin of quiescent fibroblasts/stellate cells during recovery subsequent to an inflammatory process. Previously, Elsässer et al. [19] have shown the proliferation of fibroblast-like cells in the pancreas after induction of acute pancreatitis by infusion of supramaximal doses of cerulein. Autoradiographic analysis using tritiated thymidine incorporation revealed an early peak of interstitial cell proliferation at about $48 \mathrm{~h}$ after the start of infusion. These data show an impact of fibroblastoid cells on pancreatic regeneration; however, they do not answer the question of the origin of these cells, as to whether they were intrapancreatic or derived from other sources.

For our approach, hematopoietic eGFP-chimeric rats were generated by BM transplantation. The medial incidence of eGFP-positive leukocytes in the peripheral blood of chimeric animals was about $71 \%$ and was stable between 8 weeks and 12 months after transplantation. These results suggest that the donor BM engrafted successfully and a stable chimerism of the hematopoietic system was achieved. We analyzed BM-derived PSCs by means of multiphoton microscopy detecting eGFP in excorporated fresh pancreatic tissue. This method revealed green fluorescence in mostly clustered interstitial cells, in otherwise unstained tissue, in chimeric rats, but did not reveal whether these were PSCs or tissue-resident macrophages.

Isolation of PSCs from chimeric animals and subsequent analysis of known markers such as lipid droplets, the expression of desmin and $\alpha$-SMA, confirmed eGFP- 
A

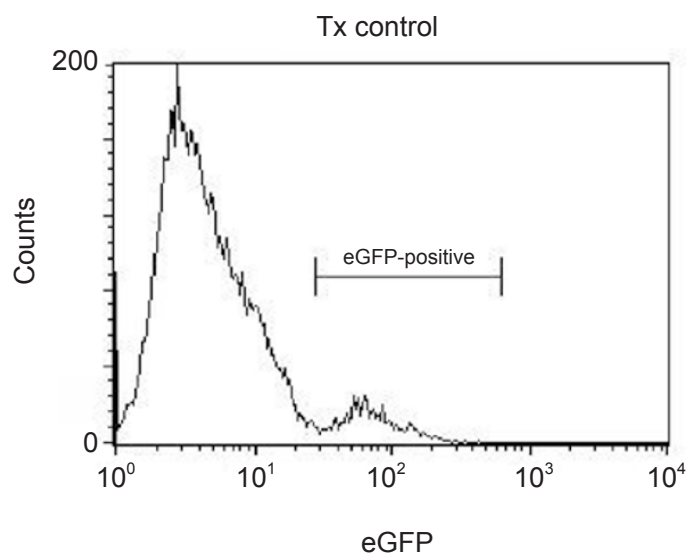

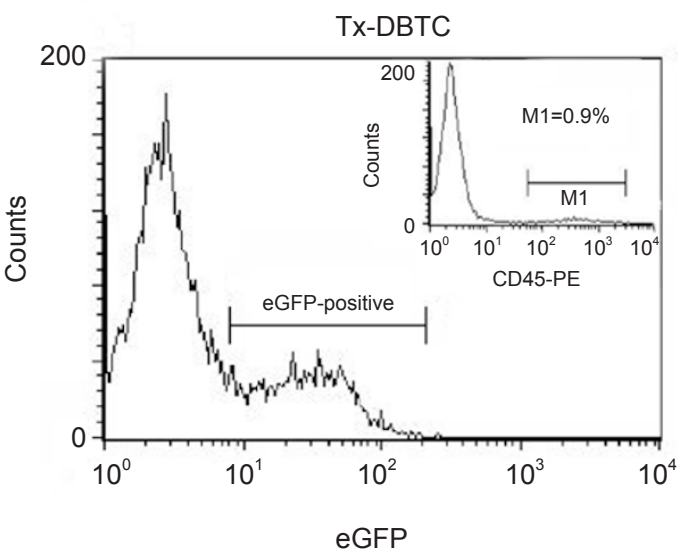

eGFP

B

\begin{tabular}{|c|c|}
\hline \multicolumn{2}{|c|}{ eGFP-positive PSC (\%) } \\
\hline $\begin{array}{c}\text { Tx control }(n=10) \\
(\text { mean } \pm \text { SEM })\end{array}$ & $\begin{array}{c}\text { Tx-DBTC }(n=10) \\
(\text { mean } \pm \text { SEM })\end{array}$ \\
\hline $6.9 \pm 2.7$ & $18.3 \pm 8.2^{*}$ \\
\hline
\end{tabular}

$* P=0.05$

Figure 6 FACS analysis of eGFP-positive PSCs. Cells were obtained from the pancreas of Tx controls and of DBTC-induced pancreatitis followed by regeneration. Isolation of PSCs has been performed at 4 weeks after the application of $4 \mathrm{mg}$ per $\mathrm{kg}$ body weight of DBTC followed by cultivation for 5 days, including one passage. The adherent growing PSCs were detached from the culture plates using trypsin-EDTA and subjected to flow cytometry detecting eGFP fluorescence in Fl1. (A) Representive histograms of FACS analysis. EGFP-positive PSCs (7\%) isolated from chimeric controls were detected, whereas PSCs from TX-DBTC contained $21 \%$ eGFP-positive cells, the insert shows that only about $1 \%$ of the analyzed cells were positive for the common leukocyte antigen CD45. (B) Evaluation of FACS data for eGFP-positive PSCs obtained from Tx controls ( $n$ $=10)$ and Tx-DBTC $(n=10)$. The rate of eGFP-positive cells in the Tx-DBTC group was threefold higher compared to the Tx controls. Results are expressed as mean \pm SEM, ${ }^{*} P=0.05$.

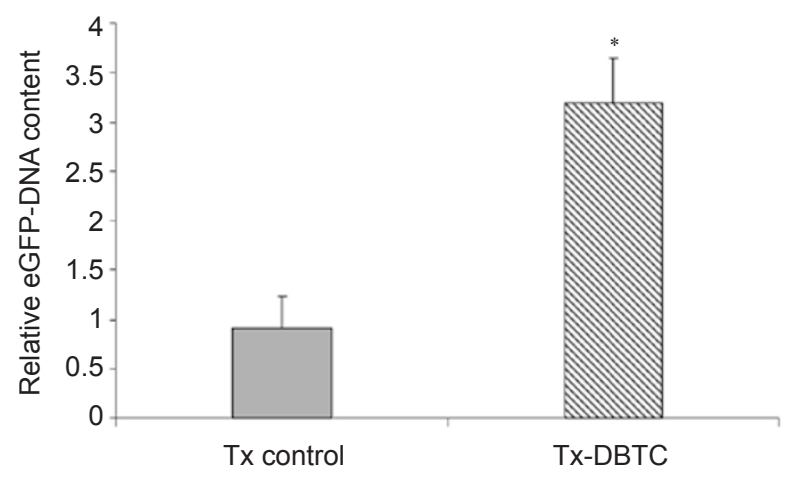

Figure 7 Evaluation of data obtained from real-time PCR for the eGFP gene in PSCs. Cells were obtained from Tx controls and from regenerated pancreas (Tx-DBTC). Isolation of PSCs from regenerated pancreas has been performed 4 weeks after the application of $4 \mathrm{mg}$ per $\mathrm{kg}$ body weight of DBTC followed by cultivation for 7 days including one passage. Genomic DNA was extracted and subjected to the TaqMan assay. Results are given as mean \pm SEM; Tx control, $n=8$ and Tx-DBTC, $n=14 ; * P=0.002$. expressing cells to be resident quiescent PSCs. In addition, the existence of eGFP in isolated PSCs could be shown by flow cytometry and by the assessment of the eGFP gene by real-time PCR. Using FACS analysis, about 7\% eGFP-positive cells were found in PSCs obtained from transplanted animals. Even though a mild transient inflammatory reaction in response to the irradiation before transplantation cannot be excluded, the detection of eGFP-positive PSCs in rats up to 12 months after irradiation and transplantation strongly supports a constitutive migratory movement of BM cells into peripheral organs followed by differentiation processes, finally resulting in tissue-specific cell populations.

The model of pancreatic regeneration following DBTC-induced acute pancreatitis was used to investigate the contribution of BM to PSC regeneration. Previously, we have characterized the cell infiltration in the model of acute DBTC pancreatitis [17]. The inflammatory parameters returned to almost normal levels within 2 weeks 
after reaching a maximum at day 7 . Here, we could demonstrate a similar time course for the expression of the intermediate filament desmin and of $\alpha$-SMA. Desmin has been shown to be a specific stellate cell marker in the pancreas and the increase of desmin-positive cells may indicate PSC proliferation and/or migration $[1,2]$. The generation of $\alpha$-SMA stress fibers defines the formation of the myofibroblastic phenotype associated with functional cell activation $[3,16]$. The correlation between leukocyte infiltration and PSC activation in the DBTC-induced model of acute pancreatitis was in agreement with numerous reports showing PSC stimulation by inflammatory reactions $[20,21]$. The restoration process requires the deletion of cells that infiltrated and/or proliferated within the time of acute injury. The mechanisms of this 'tissue cleaning' are not fully understood; however, results generated by our group and others suggest that the apoptotic cell death may be substantially involved [4, 22].

The purification procedure for PSCs based on the lipid content ensured the isolation of non-activated, quiescent PSCs from regenerated pancreas following DBTC-induced inflammation in chimeric rats (Tx-DBTC). Different eGFP-detection methods provided evidence that the portion of BM-derived PSCs increased about threefold during the restoration process, arguing for the stimulated immigration of BM-derived cells, and subsequent differentiation into PSCs of a quiescent state. The inflamed pancreas creates a microenvironment containing among other effective substances chemokines, which are known to promote cell migration. Using a mouse model, Seki et al. [18] have demonstrated an essential role of CCR2 on hepatic stellate cells for pathophysiological mechanisms in the liver. Our data showing the expression of CCR2 in quiescent PSCs support the hypothesis about the responsiveness of progenitor cells to chemotactic signals derived from the pancreas.

Our findings concerning the migration and differentiation of BM cells in the pancreas are supported by recent data of Marrache et al. [15]. However, in contrast to our experimental model, which focused on tissue that has recovered from a relatively short-lived inflammatory reaction, these authors investigated the effects of severe chronic pancreatic damage induced by repeated injections of supramaximal doses of cerulein for 45 weeks into a mouse xenograft model using BM cells expressing eGFP. They have shown that roughly $5 \%$ of PSCs were BM-derived. Although these cell numbers are less than that in our regeneration model, the differences in injury protocol, animal species and quantification methodology for BM-derived PSCs used by these authors could account for the quantitative difference. Interestingly, in agreement with our observations, BM-derived acinar or islet cells could not be detected in the mouse model, and ductal cells were eGFP-positive only to a marginal extent [15]. These results suggest that BM cells do not contribute substantially to the replenishment of pancreatic epithelial cells after injury. So far, it remains unclear as to what signals are necessary to initiate the mobilization and cell migration from the BM, leading BM cells to permanently abandon the place of their origin [23, 24]. The idea of circulating pluripotent cells representing an 'emergency' population, which could be available on site in case of demand, is a tempting concept. Already in 1994, Bucala et al. [25] have identified a distinct leukocyte subpopulation, termed fibrocytes, exhibiting characteristics of both stem cells and myo-/fibroblasts. Various studies have confirmed the contribution of circulating fibrocytes to wound repair and fibrosis [26, 27]. Mesenchymal stem cells represent a further BM-derived population with multilineage potential and their transition into myofibroblasts has been shown [24, 28]. A further possibility is that cell plasticity and migration between different organs might represent a constitutive phenomenon persisting during the whole life.

In summary, we have shown for the first time the BM origin of isolated quiescent PSCs. Our data demonstrate $\mathrm{BM}$ as a source of resting PSCs in normal pancreas and suggest that the migration of PSC progenitors was stimulated by signals derived from the injured/regenerating pancreas, resulting in increased numbers of BM-derived quiescent PSCs after restoration. Further experiments are necessary to identify the progenitor population(s) and the factors that control the mobilization and emigration of BM-derived cells, as well as the mechanisms for the recruitment and differentiation of progenitor cells in peripheral organs.

\section{Materials and Methods}

\section{Reagents}

Reagents were purchased from the following sources: Histopaque 1083, monoclonal mouse antibodies directed against desmin, $\alpha$-SMA, as well as an $\alpha$-SMA-Cy 3 antibody, Hoechst 33258, fuchsin, sodium nitrite, levamisole, naphtol-bis-phosphate, DBTC, oil red O and standard laboratory chemicals from Sigma (Deisenhofen, Germany); Iscove's modified Dulbecco's medium (IMDM), RPMI 1640 without phenol red, accutase from PAA (Cölbe, Germany); non-essential amino acids, penicillin, streptomycin, trypsin, superscript II reverse transcriptase, oligo (dT) $)_{12-18}$ primer from Invitrogen (Karslruhe, Germany); Nycodenz from AXIS SHIELD PoC AS (Oslo, Norway); collagenase P from Roche (Mannheim, Germany); fetal calf serum (FCS) from Biochrom (Berlin, Germany); AlexaFluor $555 \mathrm{~F}(\mathrm{ab})_{2}$ fragment goat anti-mouse or anti-rabbit from Molecular Probes (Eugene, OR, USA); monoclonal rat-specific anti-CD45-PE antibody from 
Acris (Hiddenhausen, Germany); anti-mouse IgG and monoclonal mouse APAAP complex from DAKO (Glostrup, Denmark). DNA isolation kit (innuPREP DNA Mini Kit) from Analytik Jena (Jena, Germany); TaqMan assays for real-time PCR from Applied Biosystems (Foster City, USA); BD FACS lysing solution, cell strainer from Becton Dickinson Labware (NJ, USA); RNeasy Mini RNA extraction kit, Taq Polymerase from Qiagen (Hilden, Germany); Immobilon-P (PVDF) membrane (Millipore, Bedford, MA, USA); rabbit anti-ERK (MAP kinase 42/44) antibody (Cell Signaling Technology, Beverly, MA, USA); ECL plus kit, horseradish peroxidase-labeled anti-rabbit or anti-mouse Ig antibody from Amersham (Freiburg, Germany); 12-mm diameter microscope cover glasses (Marienfeld, Lauda-Königshofen, Germany); BN/LEW hybrid were obtained from Charles River (Sulzfeld, Germany), transgenic LEW rats expressing eGFP under the control of the ubiquitin $\mathrm{C}$ promoter using a lentiviral vector construct, generated by Reichardt and co-workers [29], were propagated and generously provided by Manfred Lehmann (Institute of Biochemistry and Molecular Biology, University of Rostock, Rostock, Germany).

\section{Animal models}

Hematopoietic chimerism All animal work was carried out as per the procedural and ethical guidelines of the local Animal Care and Use Committee.

Female BN/LEW hybrid rats received a whole-body irradiation of 7.5 Gy using Cesium 137 radiation source. The following day, the BM of 6-week-old female eGFP transgenic LEW rats was obtained by flushing the medullary cavities of femurs. Marrow cells were filtered, washed and checked for viability using the trypan blue method. $4 \times 10^{7}$ cells per $\mathrm{ml}$ were injected into each irradiated $\mathrm{BN} / \mathrm{LEW}$ rat via the tail vein. All animals received water containing sulfamethoxazol $(0.5 \mathrm{mg} / \mathrm{ml})$, trimethoprim $(0.01 \mathrm{mg} / \mathrm{ml})$, tetracycline $(0.36 \mathrm{mg} / \mathrm{ml})$ and neomycin $(0.58 \mathrm{mg} / \mathrm{ml})$ for 2 weeks [30].

DBTC-induced model of pancreas regeneration DBTC has been shown to initiate dose- and strain-dependent pancreatic inflammatory reactions in rats $[17,31]$. Chimeric $\mathrm{BN} / \mathrm{LEW}$ rats responded to 4-6 mg per kg body weight of DBTC with an acute interstitial pancreatitis followed by pancreatic regeneration during 4 weeks.

After reaching a stable hematopoietic chimerism detected by FACS analysis of the peripheral blood cells ( 8 weeks), 38 transplanted rats were subjected to the DBTC-mediated model of pancreatic regeneration. For this approach, a respective amount of DBTC was applied as described previously [31]. Briefly, DBTC was dissolved in a mixture of glycerol/ethanol (3:2) followed by injection into the tail vein in a maximum volume of $200 \mu \mathrm{l}$ per animal.

At the indicated time points after DBTC administration, animals were killed under pentobarbital anaesthesia by cervical dislocation. The pancreas was subjected to the particular procedure appropriate for subsequent investigations.

\section{Immunohistochemical characterization of PSCs in DBTC- induced pancreatitis \\ The phenotype of PSCs in the time course of pancreatitis in- duced by $4 \mathrm{mg}$ per $\mathrm{kg}$ body weight of DBTC was characterized using immunohistochemical detection of desmin and $\alpha$-SMA. For this approach, the pancreas was removed 3, 7, 14 and 28 days after}

DBTC treatment, shock-frozen and stored in liquid nitrogen until use. Three animals were analyzed per day.

Antibody labeling was performed on $5 \mu \mathrm{m}$ cryostat sections by means of the alkaline phosphatase anti-alkaline phosphatase technique (APAAP). In brief, the specimens were incubated sequentially with unlabeled primary mouse monoclonal antibody against desmin and $\alpha$-SMA, rabbit anti-mouse IgG and monoclonal mouse APAAP complex. Alkaline phosphatase activity was visualized by incubating the specimens with a substrate containing new fuchsin, sodium nitrite, levamisole and naphtol-bis-phosphate according to Emmrich et al. [32]. Cell nuclei were counterstained with Mayer's hematoxylin and mounted in glycogel. The number of positively stained cells was given in cells per $\mathrm{mm}^{2}$.

\section{Detection of eGFP-positive cells in the pancreas by mul- tiphoton microscopy}

For multiphoton analysis, freshly excorporated unfixed tissue was placed in a tissue culture dish and submerged in $\mathrm{CO}_{2}$-independent medium containing $20 \%$ FCS, kept at $37{ }^{\circ} \mathrm{C}$ throughout the procedure. Analysis was carried out using a TriMScope (LaVision BioTec, Bielefeld, Germany) equipped with a Chameleon TitanSapphire laser (Coherent Inc, CA, USA) with maximum output at $800 \mathrm{~nm}$ wavelength connected to a Zeiss fixed-stage microscope equipped with a $\times 40$ water-immersion objective. Tissue was scanned with 64 parallel laserbeams and confocal signals passing a 535/20 nm bandpass filter were detected using a CCD camera. Micrographs represent projections of optical slices of roughly 25 $\mu \mathrm{M}$ in depth.

\section{PSC isolation and cultivation}

Due to the vastly different fluorescence intensity of indirect immunostaining and endogenous eGFP, the reliability of quantification of co-localized signals in tissue samples is limited. Therefore, our experiments were focussed on isolated PSCs.

To characterize the contribution of the BM to PSCs, freshly isolated and in vitro propagated cells have been investigated. Untreated chimeric BN/LEW rats (Tx control) and animals recovered from DBTC-induced pancreatitis 4 weeks after initiation of the disease (Tx-DBTC) were used. The isolation procedure was based on the high fat content in non-activated PSCs $[1,2]$. After mechanic and enzymatic dispersion of the pancreas, stellate cells were purified by isopycnic density centrifugation as previously described [22]. Isolated PSCs were suspended in IMDM supplemented with $10 \% \mathrm{FCS}, 1 \%$ non-essential amino acids, $100 \mathrm{U} / \mathrm{ml}$ penicillin and $100 \mu \mathrm{g} / \mathrm{ml}$ streptomycin. Cell suspension was seeded into six-well culture plates and kept at $37{ }^{\circ} \mathrm{C}$ in a $5 \% \mathrm{CO}_{2}$ humidified atmosphere. PSCs adhered to the culture plate surface during the 4-h incubation period. Afterward, the supernatant was removed, adherent PSCs were washed carefully with phosphate-buffered saline (PBS) to eliminate contaminating cells and cell debris floating in the medium.

For immunofluorescence staining, isolated PSCs were seeded onto microscope cover glasses placed in 12-well culture dishes followed by the cultivation procedure, as described above.

\section{Histochemical staining of intracellular fat}

Intracellular fat droplets were visualized by oil red O staining. For this approach, three parts of an oil red O stock solution (1\% $\mathrm{wt} / \mathrm{vol}$ dissolved in isopropanol) were mixed with two parts of 
distilled water. Cells grown on glass coverslips and fixed in 3\% paraformaldehyde were incubated with the dye solution for $15 \mathrm{~min}$ followed by counterstaining using Mayer's hematoxylin.

\section{Indirect immunofluorescence staining and cLSM}

For indirect immunofluorescence staining, PSCs were cultivated on glass coverslips. At the end of the incubation time, specimens were fixed with $2.5 \%$ parafomaldehyde, permeabilized using methanol followed by incubation with the respective primary antibody. Antibody binding was detected by the secondary AlexaFluor 555 immunoglobulin directed against the species of the primary antibody. The anti- $\alpha$-SMA antibody was labeled with fluorescent probe Cy3. Nuclei were visualized using Hoechst33258 dye. Analysis of eGFP expression in isolated PSCs was carried out on cells plated on glass coverslips. Cells were fixed at the indicated time points as described above, but without methanol. For the detection of eGFP-positive cells in the pancreatic tissue, the organ was fixed in paraformaldehyde. Tissue sections $(5 \mu \mathrm{m})$ were subjected to fluorescence staining procedure, as described for isolated cells. Analysis of fluorescence was performed by cLSM (LSM 510 UV Meta, Carl Zeiss, Jena, Germany) at a pinhole diameter of 1 Airy unit or optical slice of $<1 \mu \mathrm{M}$. All analyses represented here were three dimensional, pictures representing projections of several optical slices depicting 4.5-6.5 $\mu \mathrm{M}$ of depth for cells and about $3 \mu \mathrm{M}$ of tissue depth for cryosections.

\section{Flow cytometry}

The eGFP protein in BM, peripheral blood cells as well as in PSCs has been assessed by flow cytometry measuring the F11 fluorescence in a FACScalibur (Becton Dickinson). An antibody detecting the rat leukocyte common antigen (CD45) conjugated to PE was used to identify leukocytes.

Blood $(100 \mu \mathrm{l})$ obtained from the rat tail vein were incubated with $10-\mu 1$ anti-CD45-PE for 20 min on ice. Subsequently, erythrocytes were removed using the FACS lysing solution according to the manufacturers instructions. Leukocytes were washed twice with phenol red-less RPMI 1640 and stored until FACS analysis in $0.5 \%$ paraformaldehyde at $4{ }^{\circ} \mathrm{C}$.

The expression of eGFP in isolated PSCs was determined after the first passage at different time points during in vitro cultivation. For this approach, cells were detached from culture plates by accutase according to standard protocols, washed twice with PBS (pH 7.4), fixed with $0.5 \%$ PFA. Leukocyte contaminations within the PSC cultures could be examined using CD45-PE staining. Data were evaluated using the Cellquest software (Becton Dickinson).

\section{Immunoblotting}

Protein extracts of PSCs were harvested as previously described [22]. Lysates were separated by SDS-polyacrylamide gel electrophoresis and blotted onto PVDF membrane. Blots were incubated with the respective primary antibody for $2 \mathrm{~h}$ at room temperature. For visualization of the antibody binding, filters were exposed to a horseradish peroxidase (POD)-labeled anti-rabbit or anti-mouse Ig antibody and developed using the ECL Plus kit.

\section{Reverse transcription - polymerase chain reaction}

Analysis of mRNA expression has been performed as described previously [17]. In brief, total RNA was isolated using the RNeasy kit according to the manufacturers instructions followed by tran- scription into cDNA. Relative quantification of mRNA expression was performed by using competitive PCR with a synthetic DNA fragment as internal standard for the housekeeping gene hypoxanthine phosphoribosyl transferase (HPRT). On the basis of the different amplicon sizes of cDNA and standard, PCR products could be separated electrophoretically in an ethidium bromide-containing agarose gel. On the basis of the ethidium bromide fluorescence intensity reflecting HPRT expression, various cDNA samples were adjusted to equal input concentrations.

\section{Detection of eGFP transgene-containing cells}

A duplex real-time PCR assay was employed for the relative quantification of eGFP transgene copies in PSCs from the chimeric rats. Therefore, a VIC/TAMRA TaqMan assay was designed for the eGFP target sequence, employing forward primer: 5'-GTC CGC CCT GAG CAA AGA-3'; reverse primer: 5'-TCA CGA ACT CCA GCA GAC C-3'; probe: 5'-CCC AAC GAG AAG CGC GAT CAC-3'. In a duplex reaction using a commercially available FAM6 assay for the single copy gene RNaseP (Applied Biosystems, Foster City, USA), the $\mathrm{C} t$ values were used for relative estimation of the copy number.

PSCs were obtained from Tx controls and from regenerated pancreas (Tx-DBTC). Cell isolation has been performed at 4 weeks after the application of $4 \mathrm{mg}$ per $\mathrm{kg}$ body weight of DBTC followed by cultivation for 7 days, including one passage. Genomic DNA was extracted using the innuPREP DNA kit (Analytik Jena) according to the manufacturers instructions.

\section{Statistical analysis}

Data are expressed as mean \pm SEM. Statistical significance of differences was determined using the Mann-Whitney Rank Sum Test at a minimum $P \leq 0.05$.

\section{Acknowledgments}

The authors thank Edith Prestin and Katrin Sievert (Univeristy of Rostock) for the excellent technical assistance as well as for the help with the animal experiments.

\section{References}

1 Apte MV, Haber PS, Applegate TL, et al. Periacinar stellate shaped cells in rat pancreas: identification, isolation, and culture. Gut 1998; 43:128-133.

2 Bachem MG, Schneider E, Gross H, et al. Identification, culture, and characterization of pancreatic stellate cells in rats and humans. Gastroenterology 1998; 115:421-432.

3 Jaster R. Molecular regulation of pancreatic stellate cell function. Mol Cancer 2004; 3:1-8.

4 Klonowski-Stumpe H, Fischer R, Reinehr R, Luthen R, Haussinger D. Apoptosis in activated rat pancreatic stellate cells. Am J Physiol Gastrointest Liver Physiol 2002; 283:G819-G826.

5 Ramiya VK, Maraist M, Arfors KE, Schatz DA, Peck AB, Cornelius JG. Reversal of insulin-dependent diabetes using islets generated in vitro from pancreatic stem cells. Nat Med 2000; 6:278-282.

6 Hess D, Li L, Martin M, Sakano S, et al. Bone marrow-de- 
rived stem cells initiate pancreatic regeneration. Nat Biotechnol 2003; 21:763-770.

7 Dor Y, Brown J, Martinez OI, Melton DA. Adult pancreatic beta-cells are formed by self-duplication rather than stem-cell differentiation. Nature 2004; 429:41-46.

8 Sumi S, Gu Y, Hiura A, Inoue K. Stem cells and regenerative medicine for diabetes mellitus. Pancreas 2004; 29:e85-e89.

9 Jensen JN, Cameron E, Garay MV, Starkey TW, Gianani R, Jensen J. Recapitulation of elements of embryonic development in adult mouse pancreatic regeneration. Gastroenterology 2005; 128:728-741.

10 Yalniz M, Pour PM. Are there any stem cells in the pancreas? Pancreas 2005; 31:108-118.

11 Mato E, Lucas M, Petriz J, Gomis R, Novials A. Identification of a pancreatic stellate cell population with properties of progenitor cells: new role for stellate cells in the pancreas. Biochem J 2009; 421:181-191.

12 Russo FP, Alison MR, Bigger BW, et al. The bone marrow functionally contributes to liver fibrosis. Gastroenterology 2006; 130:1807-1821.

13 Lama VN, Phan SH. The extrapulmonary origin of fibroblasts: stem/progenitor cells and beyond. Proc Am Thorac Soc 2006; 3:373-376.

14 van Amerongen M, Bou-Gharios G, Popa E, et al. Bone marrow-derived myofibroblasts contribute functionally to scar formation after myocardial infarction. J Pathol 2008; 214:377-386.

15 Marrache F, Pendyala S, Bhagat G, Betz KS, Song Z, Wang TC. Role of bone marrow-derived cells in experimental chronic pancreatitis. Gut 2008; 57:1113-1120.

16 Sparmann G, Hohenadl C, Tornoe J, et al. Generation and characterization of immortalized rat pancreatic stellate cells. Am J Physiol Gastrointest Liver Physiol 2004; 287:G211G219.

17 Hense S, Sparmann G, Weber H, Liebe S, Emmrich J. Immunologic characterization of acute pancreatitis in rats induced by dibutyltin dichloride (DBTC). Pancreas 2003; 27:e6-e12.

18 Seki E, de Minicis S, Inokuchi S, et al. CCR2 promotes hepatic fibrosis in mice. Hepatology 2009; 50:185-197.

19 Elsasser HP, Adler G, Kern HF. Time course and cellular source of pancreatic regeneration following acute pancreatitis in the rat. Pancreas 1986; 1:421-429.

20 Luttenberger T, Schmid-Kotsas A, Menke A, et al. Platelet- derived growth factors stimulate proliferation and extracellular matrix synthesis of pancreatic stellate cells: implications in pathogenesis of pancreas fibrosis. Lab Invest 2000; 80:4755.

21 Mews P, Phillips P, Fahmy R, et al. Pancreatic stellate cells respond to inflammatory cytokines: potential role in chronic pancreatitis. Gut 2002; 50:535-541.

22 Sparmann G, Glass A, Brock P, et al. Inhibition of lymphocyte apoptosis by pancreatic stellate cells: impact of interleukin-15. Am J Physiol Gastrointest Liver Physiol 2005; 289:G842-G851.

23 Alison MR, Poulsom R, Otto WR, et al. Plastic adult stem cells: will they graduate from the school of hard knocks? $J$ Cell Sci 2003; 116:599-603.

24 Direkze NC, Jeffery R, Hodivala-Dilke K, et al. Bone marrow-derived stromal cells express lineage-related messenger RNA species. Cancer Res 2006; 66:1265-1269.

25 Bucala R, Spiegel LA, Chesney J, Hogan M, Cerami A. Circulating fibrocytes define a new leukocyte subpopulation that mediates tissue repair. Mol Med 1994; 1:71-81.

26 Abe R, Donnelly SC, Peng T, Bucala R, Metz CN. Peripheral blood fibrocytes: differentiation pathway and migration to wound sites. J Immunol 2001; 166:7556-7562.

27 Barth PJ, Ebrahimsade S, Hellinger A, Moll R, Ramaswamy A. CD34 $4^{+}$fibrocytes in neoplastic and inflammatory pancreatic lesions. Virchows Arch 2002; 440:128-133.

28 Jiang Y, Jahagirdar BN, Reinhardt RL, et al. Pluripotency of mesenchymal stem cells derived from adult marrow. Nature 2002; 418:41-49.

29 van den Brandt J, Wang D, Kwon SH, Heinkelein M, Reichardt HM. Lentivirally generated eGFP-transgenic rats allow efficient cell tracking in vivo. Genesis 2004; 39:94-99.

30 Wolff D, Frei E, Hofmeister N, et al. Methotrexate-albumin and aminopterin-albumin effectively prevent experimental acute graft-versus-host disease. Transplantation 2006; 82:527-533.

31 Sparmann G, Merkord J, Jaschke A, et al. Pancreatic fibrosis in experimental pancreatitis induced by dibutyltin dichloride. Gastroenterology 1997; 112:1664-1672.

32 Emmrich J, Weber I, Nausch M, et al. Immunohistochemical characterization of the pancreatic cellular infiltrate in normal pancreas, chronic pancreatitis and pancreatic carcinoma. $\mathrm{Di}$ gestion 1998; 59:192-198. 